\title{
ONE-SHOT SEGMENTATION OF BREAST, PECTORAL MUSCLE, AND BACKGROUND IN DIGITISED MAMMOGRAMS
}

\author{
Arnau Oliver, Xavier Lladó, Albert Torrent, and Joan Martí \\ Dept. of Computer Architecture and Technology \\ University of Girona \\ 17071, Girona, Spain \\ \{aoliver,llado,atorrent,joanm\}@ eia.udg.edu
}

\begin{abstract}
The segmentation of the breast from the background and the pectoral muscle is the first pre-processing step in computerised mammographic analysis. This problem is usually solved by dividing it into two different segmentation strategies, one for the background and another one for the pectoral muscle. In this paper we tackle this problem jointly using a supervised single strategy. Namely, from a set of manually segmented mammograms, we model each of the three regions (breast, pectoral muscle, and background) using position, intensity, and texture information. Although the approach requires a training step, it allows a fast and reliable segmentation of new mammograms. The obtained results using 149 mammograms of the MIAS database show a high degree of overlap between manual and automatic segmentation.
\end{abstract}

Index Terms - Medical Imaging, Breast Segmentation, Computer Aided Diagnosis, Atlas, Texture.

\section{INTRODUCTION}

Breast cancer is still a worldwide health problem and screening programs are promoted in each country to detect cancer in its early stages, a crucial step for improving prognosis. Mammography is the most reliable technique to detect abnormalities and automatic tools are being developed to assist radiologists in their everyday current practice $[1,2]$. The first step of these algorithms is the localisation of the breast area inside the whole mammogram. As shown in Figure 1, mammograms contain not only the breast area but also a portion of the pectoral muscle and background, which may be (very) noisy and even may contain labels.

Although breast area segmentation is not a new research topic [3], no single method has emerged as a standard approach and new proposals are still appearing to deal with this problem $[4,5]$. Most of these works are unsupervised and based on region or edge information. However, mammograms acquired from different manufacturer machines or

This work was partially funded by the Spanish R+D+I grant TIN201237171-C02-01. even with the same machine but using different acquisition parameters may present very different characteristics, needing to readjust the parameters of each algorithm. In contrast, in this paper we present a supervised algorithm for breast segmentation, a more natural way to deal with inter-database variability. Besides, it allows to tackle the segmentation in a one-shot strategy, oppositely to the vast state of the art algorithms, where one approach was used for the segmentation of the background (also referred as skin-line segmentation) and another one for the segmentation of the pectoral muscle.

Based on a training set of mammograms manually segmented by an expert into background, breast, and pectoral muscle, we model each pixel of the region using a probabilistic model which merges the intensity, texture, and position estimated from that region. Subsequently, mammograms of the testing set are segmented pixel-wise using this approach. Finally, in order to remove isolated bad classified pixels, postprocessing techniques are applied to provide the final segmentation result. The evaluation is performed following a crossvalidation scheme and using the MIAS database.

\section{BREAST AREA SEGMENTATION}

We model the probability $P$ of a pixel $p$ to belong to background, breast, or pectoral muscle according to its position, intensity, and texture information $(p(x, i, t))$. Next subsections explain in more detail how these models are computed and finally combined.

\subsection{Atlas information}

Mammograms are always located in a similar manner: the pectoral muscle in the upper corner of the image, the breast region surrounding the pectoral muscle, and the background surrounding the breast. Hence, position information should play a key role when segmenting mammograms. However, this information has not properly been exploited in most of the previous algorithms. To include a spatial probability into the algorithm, we build three different atlases, one for each 


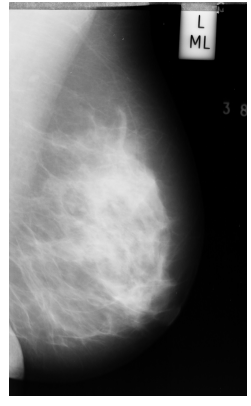

(a)

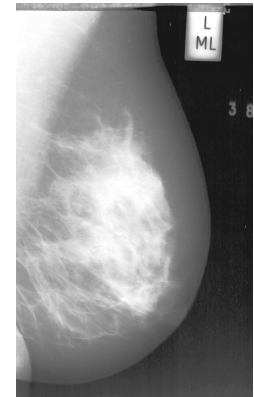

(b)

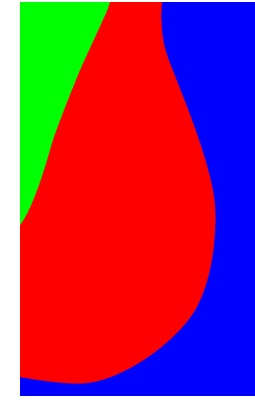

(c)
Fig. 1. Breast segmentation. (a) mammogram, (b) enhanced mammogram, and (c) manual segmentation. Notice in image (b) that the breast area is larger that it is visually shown in the original image and that the background is noisy and may contain some annotations.

region. All images from a training set, manually segmented in the three regions, are superposed to the same origin. Two main problems arise in this procedure. Firstly, there are mammograms of different sizes, and secondly, the mammograms could have different orientation if they are left or right views.

To solve the first problem, we use mammograms of the same size. Although this may seem too restrictive, this is not the case since, usually, mammograms are acquired just in two / three sizes, depending on the size of the breast. Notice that a reshape of the images would result in incongruents results, since in small images the breast may cover almost all of the image, while in large images, the breast just covers part of it. On the other hand, the second problem can be solved by flipping all the images coming from right (or left) breasts. If this information is not available, a possible solution is looking for the centre of masses of the image. If it is larger than the mammogram half-width, the image should be flipped.

Therefore, the probability density function $A$ for each pixel location $(x)$ to belong to region $R$ (being background, breast, and pectoral muscle) is computed as:

$$
A_{R}(x)=\frac{n(x \in R)}{N}
$$

where $n(x \in R)$ represents the number of locations $x$ being in region $R$, and $N$ the total number of images of the training set. First row of Figure 2 shows the three atlases obtained, where red pixels correspond to higher probabilities.

Notice that usually, a registration process is needed when constructing an atlas. In our approach we are intrinsically assuming that the images are "similar enough" to construct the atlas. Actually, the main differences in the atlas are due to size differences, and larger size differences are already avoided due to the fact that we are using mammograms of the same size. Besides, notice that this step is prior to breast registration and indeed registration approaches assume this step is already done.

\subsection{Intensity information}

The pectoral muscle and the background region usually present characteristic intensity values. The pectoral muscle tends to be brighter than the breast, although intensities may be similar with the ones of the internal dense areas. In contrast, background is usually darker than the breast, except by the fact that it may contain some labels. In our approach, intensity information of background, breast, and pectoral muscle areas is encoded using the histogram of each of these areas computed over all the images of the training set. Once the histogram is computed it is normalised so that the sum of the histogram is equal to 1 . This histogram actually shows the intensity distribution of each region. To define the probability $I_{R}$ of each intensity grey-value to belong to each region $R$, we combine the histograms as:

$$
I_{R}(i)=\frac{H_{R}(i)}{\left.\sum_{j=1}^{3} H_{j}(i)\right)}
$$

The result of this equation represents the probability distribution for each grey-level value to belong to background, breast, and pectoral muscle. Notice that if there is an intensity not represented in the training set the result of this equation is undetermined. We avoid this issue assuming a uniform distribution for each class. Second row of Figure 2 shows the probability of each pixel of a mammogram to be pectoral muscle, breast, or background just using intensity information. As expected, dense areas of the mammogram and also the labels are considered as pectoral muscle just taking intensity information into account.

\subsection{Texture information}

Texture information represents the relation between a pixel and its neighbourhood. It is assumed that different regions should contain a different texture. In this work we use local binary patterns (LBP) [6] to obtain this information, which has been successfully used for a variety of applications including mammography $[7,8]$. Each pixel of the image is converted to a binary value obtained when thresholding its neighbourhood using the own intensity as a threshold. The neighbours with higher or equal intensity are assigned to one, while the ones with lower intensity are converted to zero. Subsequently, the neighbourhood is converted to a binary value by concatenating those 0 and 1 . Finally, this binary value is converted to its decimal value.

To characterise each region, we labelled all the pixels with the corresponding LBP codes and the histogram of the labels were computed as the texture descriptor of that region. Similarly to the intensity information, the histogram was computed with the regions of the training images. In the same way, the probability of each LBP code to belong to background, breast, or pectoral muscle was computed as: 

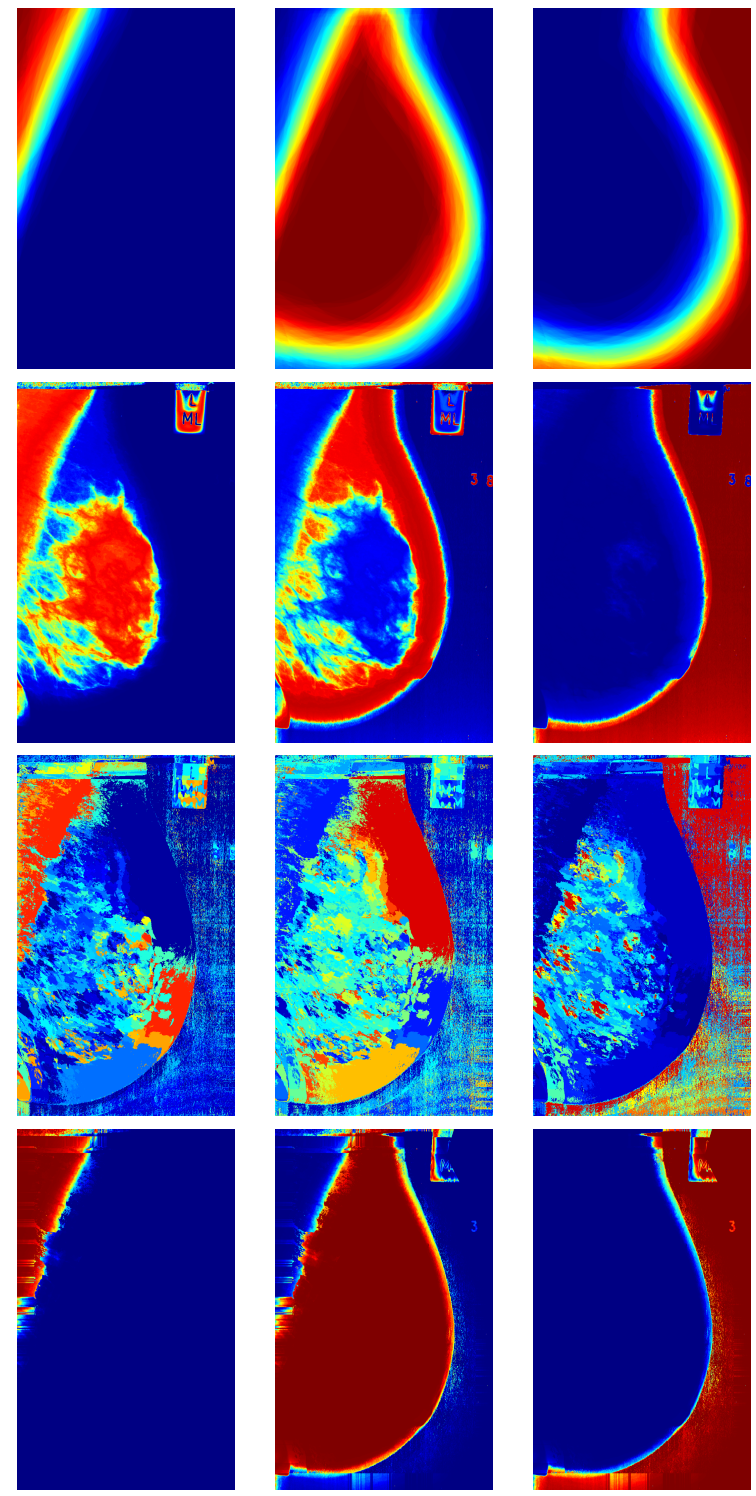

(a)
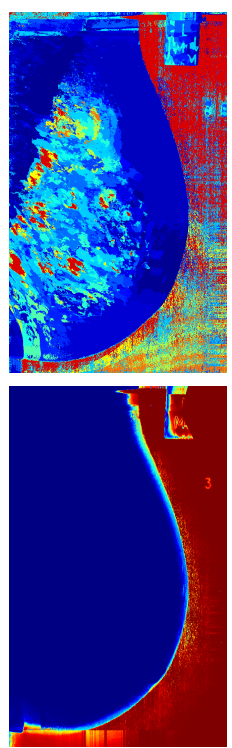

(c)

Fig. 2. Result of each step of the probabilistic model applied to the mammogram shown in Figure 1 (red colour, high probability; blue colour, low probability). (a) shows the pectoral modelling, (b) the modelling of the breast region, and (c) the modelling of the background. From upper to bottom row, atlas probability, intensity probability, texture probability, and final normalised probability.

$$
T_{R}(t)=\frac{L B P_{R}(i)}{\left.\sum_{j=1}^{3} L B P_{j}(i)\right)}
$$

where LBP represents the LBP histogram of codes. Third row of Figure 2 shows the result of modelling each pixel of the mammogram using texture information. Texture information allows to discriminate better fatty regions near the skin-line.
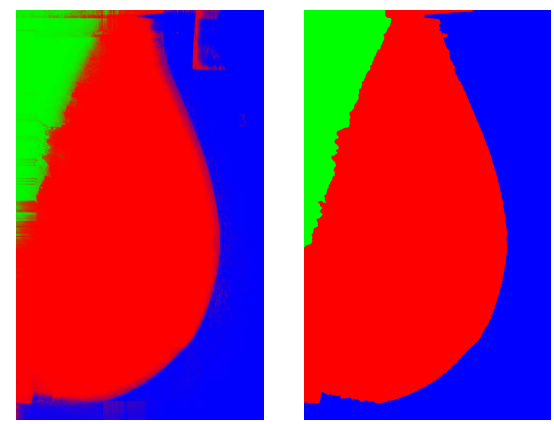

Fig. 3. Segmentation result of the mammogram shown in Figure 1 before and after the post-processing step.

\subsection{Probabilistic model}

To segment a new mammogram the three sources of information above mentioned are combined. It is commonly assumed that these three values are independent to each other $[9,10]$. Hence, for each region, we can compute its probability function as:

$$
P_{R}(p(x, i, t))=A_{R}(x) I_{R}(i) T_{R}(t)
$$

where the probability of each pixel to belong to each region $R$ (breast, background, pectoral) is characterised by the probability distributions of position $A_{R}$, intensity $I_{R}$, and texture $T_{R}$. When multiplying the three probabilities we obtain, for each pixel, the likelihood to belong to each region. Subsequently, the three probabilities for each pixel are normalised by they sum, obtaining the final probabilistic result shown in last row of Figure 2. Notice that atlas, intensity, and texture give different but complementary information. The final step is to assign each pixel to the class with higher probability.

\subsection{Post-processing}

The result may contain some pixels or small regions badly classified (notice in Figure 3 that part of the label in the background is classified as breast). In order to refine these problems we included a final post-processing step. Again, the pixels belonging to pectoral muscle, breast area, and background are divided into three different images, one per class. An erosion operation with a round structural element of size 5 and subsequently a dilation operation with a round structural element of size 3 are applied to each image. This allows to remove small regions linked by few pixels to the largest area of each image, which is automatically located by using a component labelling algorithm. When combining the three images into the final segmentation, some regions without labelling may appear, which are then filled by looking the class of each nearest labelled neighbours, obtaining the final segmentation shown in Figure 3. 
Table 1. Obtained Dice coefficients when using only atlas information (A), intensity information (I), texture information (T), and the different combinations (atlas+intensity, atlas+texture, intensity+texture, and the all three sources) for breast, pectoral muscle, and background.

\begin{tabular}{|c|c|c|c||c|c|c||c|}
\hline \multicolumn{1}{l|}{} & A & I & T & A+I & A+T & I+T & All \\
\hline Br. & 0.89 & 0.81 & 0.75 & 0.95 & 0.95 & 0.86 & 0.97 \\
\hline P. & 0.73 & 0.50 & 0.37 & 0.83 & 0.79 & 0.54 & 0.83 \\
\hline Bk. & 0.82 & 0.90 & 0.64 & 0.93 & 0.93 & 0.93 & 0.96 \\
\hline
\end{tabular}

\section{RESULTS}

The results were obtained using all the 149 images labelled as "large" of the MIAS database [11] $(4320 \times 2600$ pixels, although they were subsequently resized by a factor of 4 to speed-up the whole process). The evaluation was done using a 10-fold cross-validation scheme and the Dice similarity coefficient. In a $\mathrm{N}$-folder cross-validation all the images are divided into $\mathrm{N}$ different groups, from where $N-1$ are used to define the model, while the remaining group is used for testing. This procedure is repeated $\mathrm{N}$ times until all the groups are used for testing. Using this methodology each image appears in the test set only once. We include in the same group left and right mammograms of the same patient, in order to avoid possible bias due to the similarity between both breasts. Besides, the Dice similarity coefficient computes the overlap between the foreground (F) and the background (B):

$$
d s c=\frac{2|F \cap B|}{|F|+|B|}
$$

where the operator || refers to the number of pixels of that region. Notice that when one of the three regions is evaluated (background, breast area, pectoral muscle) the foreground is that region and the background is the union of the other two.

Table 1 shows the results of the segmentation when using the different sources of information (atlas, intensity, texture) independently, when using the combination of two, and when using all of them. The best results are obtained when all features are used together. The time used to segment a new mammogram is less than one second in a standard computer (the training was performed off-line and required around 10 minutes). Figure 4 shows qualitative results obtained with the proposed approach.

It is difficult to compare the presented approach with others approaches of the state-of-the-art. To our knowledge, this is the first proposal segmenting in a single shot the breast area from the background and the pectoral muscle. Moreover, most of the papers aiming at segmenting the background or the pectoral muscle were evaluated visually. Only the works of Wirth et al. [12] and Tzikopoulos et al. [4] presented a numerical evaluation of the segmentation of background vs the breast area and the pectoral muscle. They obtained an overlap
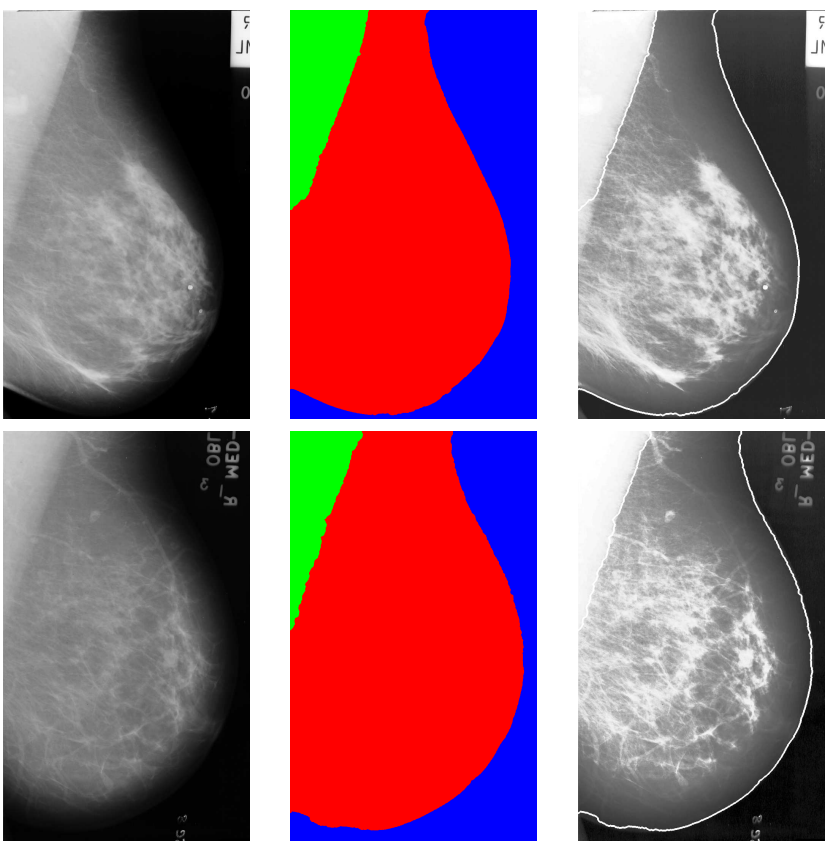

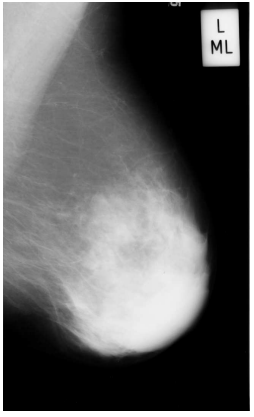

(a)

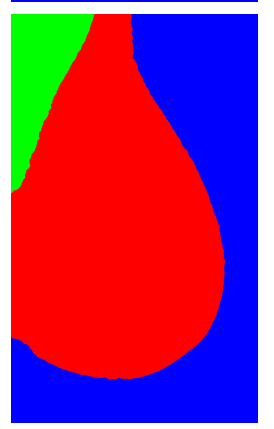

(b)

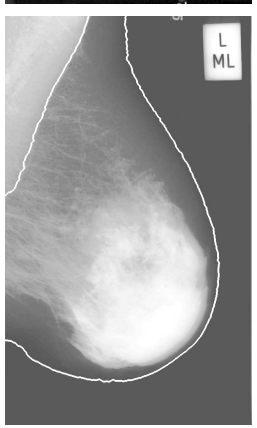

(c)
Fig. 4. Segmentation examples using the proposed approach: (a) original mammograms, (b) obtained segmentation, and (c) borders of the obtained segmentation overlaid on an enhanced representation of the mammogram.

of 0.99 , which is slightly better than the obtained in our work (0.96) when segmenting the three regions at the same time.

\section{CONCLUSIONS}

In this paper we have presented a supervised approach for one-shot segmentation of breast, pectoral muscle, and background in digitised mammograms. This step is crucial in computer aided approaches, where specific algorithms are applied only to the breast. The experimental results using the MIAS database have shown that our approach provides a fast and reliable segmentation. Further work is divided into two directions. Firstly, we want to test other probabilistic combinations in order to avoid the post-processing step, and secondly, we want to iteratively combine this approach with a registration approach for obtaining a more refined atlas estimation. 


\section{REFERENCES}

[1] J. Freixenet, A. Oliver, X. Lladó, R. Martí, J. Pont, E. Pérez, E.R.E. Denton, and R. Zwiggelaar, "Eigendetection of Masses considering False Positive Reduction and Breast Density Information," Med. Phys., vol. 35, no. 5, pp. 1840-1853, 2008.

[2] A. Oliver, A. Torrent, X. Lladó, M. Tortajada, L. Tortajada, M. Sentís, J. Freixenet, and R. Zwiggelaar, "Automatic microcalcification and cluster detection in digital and digitised mammograms," Knowl.-Based Syst., vol. 28, pp. 68-75, 2012.

[3] D. Raba, A. Oliver, J. Martí, M. Peracaula, and J. Espunya, "Breast Segmentation with Pectoral Muscle Suppression on Digital Mammograms," in Lect. Not. Comp. Sc., 2005, vol. 3523, pp. 471-478.

[4] S.D. Tzikopoulos, M. E. Mavroforakis, H.V. Georgiou, N. Dimitropoulos, and S. Theodoridis, "A fully automated scheme for mammographic segmentation and classification based on breast density and asymmetry," Comput. Meth. Prog. Biomed., vol. 102, no. 1, pp. 47-63, 2011.

[5] M. Mustra and M. Grgic, "Robust automatic breast and pectoral muscle segmentation from scanned mammograms," Signal Proc., vol. 93, no. 10, pp. 2817-2827, 2013.

[6] T. Ojala, M. Pietikäinen, and T. Mäenpää, "Multiresolution Gray-Scale and Rotation Invariant Texture Classification with Local Binary Patterns," IEEE Trans. Pattern Anal. Machine Intell., vol. 24, no. 7, pp. 971-987, 2002.

[7] A. Oliver, X. Lladó, J. Freixenet, and R. Zwiggelaar, "Classifying mammograms using texture information," in Med. Image Underst. Anal., 2007, pp. 223-227.

[8] X. Lladó, A. Oliver, J. Freixenet, R. Martí, and J. Martí, “A textural approach for mass false positive reduction in mammography," Comp. Med. Imag. Grap., vol. 33, no. 6, pp. 415-422, 2009.

[9] A. Madabhushi and D. N. Metaxas, "Combining low-, highlevel and empirical domain knowledge for automated segmentation of ultrasonic breast lesions," IEEE Trans. Med. Imag., vol. 22, no. 2, pp. 47-63, 2003.

[10] J. Massich, F. Meriaudeau, M. Sentís, S. Ganau, E. Pérez, R. Martí, A. Oliver, and J. Martí, "Automatic seed placement for breast lesion segmentation on US images," in Lect. Not. Comp. Sc., 2012, vol. 7361, pp. 308-315.

[11] J. Suckling, J. Parker, D. R. Dance, S. M. Astley, I. Hutt, C. R. M. Boggis, I. Ricketts, E. Stamatakis, N. Cerneaz, S. L. Kok, P. Taylor, D. Betal, and J. Savage, "The Mammographic Image Analysis Society Digital Mammogram Database," in Int. Work. Dig. Mammography, 1994, pp. 211-221.

[12] M. Wirth, D. Nikitenko, and J. Lyon, "Segmentation of the Breast Region in Mammograms using a Rule-Based Fuzzy Reasoning Algorithm," Int. J. Graph. Vision Image Process., vol. 5, no. 2, pp. 45-54, 2005. 